\title{
Peak reduction in OFDM using second-order cone programming relaxation
}

\author{
Marko Beko ${ }^{1,2^{*}}$, Rui Dinis 3,4 and Ramo Šendelj ${ }^{5}$
}

\begin{abstract}
In this paper, we address the problem of peak-to-average power ratio (PAPR) reduction in orthogonal frequency-division multiplexing (OFDM) systems. We formulate the problem as an error vector magnitude (EVM) optimization task with constraints on PAPR and free carrier power overhead (FCPO). This problem, which is known to be NP hard, is shown to be approximated by a second-order cone programming (SOCP) problem using a sequential convex programming approach, making it much easier to handle. This approach can be extended to the more general problem when PAPR, EVM, and FCPO are constrained simultaneously. Our performance results show the effectiveness of the proposed approach, which allows good performance with lower computational complexity and infeasibility rate than state-of-the-art PAPR-reduction convex approaches. Moreover, in the case when all the three system parameters are constrained simultaneously, the proposed approach outperforms the convex approaches in terms of infeasibility rate, PAPR, and bit error rate (BER) performances.
\end{abstract}

Keywords: Error vector magnitude; Free carrier power overhead; Orthogonal frequency-division multiplexing; Peak-to-average power ratio; Semidefinite programming; Second-order cone programming

\section{Introduction}

Orthogonal frequency-division multiplexing (OFDM) [1] are suitable for high rate transmission over severely timedispersive channels. For this reason, they were selected for broadband wireless communication systems such as digital video broadcasting (DVB) and long-term evolution (LTE). However, OFDM signals have high envelope fluctuations and high peak-to-average power ratio (PAPR), which makes them very sensitive to nonlinear effects and leads to amplification difficulties [2]. For this reason, several techniques were proposed to reduce the PAPR of OFDM signals. We can reduce the PAPR by using specially designed codes [3], but its application is limited to very specific cases and/or a small number of subcarriers. Multiple signal representations such as partial transmit sequences (PTS) techniques [4,5] and clipping techniques $[6-8,10]$ are much more flexible and suitable for OFDM signals with a large number of subcarriers.

*Correspondence: mbeko@uninova.pt

1 Universidade Lusófona de Tecnologias e Humanidades, Campo Grande, Lisbon 1749-024, Portugal

2 UNINOVA, Campus FCT/UNL, Monte de Caparica 2825-114, Portugal

Full list of author information is available at the end of the article
However, with PTS techniques, we might need to transmit side information, and the transmitter complexity can increase substantially. On the other hand, the nonlinear nature of clipping might lead to significant performance degradation. Tone reservation techniques (TR) $[9,11,12]$ are particularly interesting for large constellations since we have only a small degradation in the power and spectral efficiency (due to nondata subcarriers).

Convex optimization has recently emerged as an efficient tool for reducing the PAPR of OFDM signals [10-16]. This can be explained in part by the fact that convex optimization methods can efficiently compute global solutions to large-scale problems in polynomial time. In [15], an iterative second-order cone programming (SOCP) approach was proposed to pursue the quasi-constant PAPR value of OFDM signals. In [16], a semidefinite relaxation (SDR) technique is employed to reduce the PAPR values of OFDM symbols. Although convex optimization approaches show advantages over the classical repeated clipping and filtering (RCF) approach [7], it is important to note that they may fail to deliver feasible solution to the PAPR problem; see $[15,16]$. This is due to the fact that in $[15,16]$, the feasible (nonconvex) set of the original (nonconvex) PAPR problem lies within the feasible (convex)

\section{是 Springer}

(c) 2014 Beko et al: licensee Springer. This is an Open Access article distributed under the terms of the Creative Commons Attribution License (http://creativecommons.org/licenses/by/4.0), which permits unrestricted use, distribution, and reproduction in any medium, provided the original work is properly credited. 
set of the relaxed convex problem. Hence, the solutions provided by the methods proposed in $[15,16]$ are not necessarily feasible for the original problem. Furthermore, a complex semidefinite programming problem (SDP) is solved in [16].

To overcome these drawbacks, in this paper, we propose to minimize the error vector magnitude (EVM) subject to constraints on the PAPR and free carrier power overhead (FCPO). We introduce an efficient sequential convex programming approach to solve the corresponding nonconvex problem by rewriting the nonconvex constraints as a difference of two convex functions. We show that the new approach can also be successfully applied to the case when the PAPR, EVM, and FCPO are simultaneously constrained.

The remainder of this paper is organized as follows. The OFDM symbol design for PAPR reduction is formulated as an optimization problem in Section 2, and in Section 3, we introduce our approach for solving this optimization problem. A set of performance results is presented in Section 4, and Section 5 is concerned with the conclusions of this paper.

\section{Problem formulation}

Let $c_{0} \in \mathbb{C}^{N}$ be an original OFDM frequency-domain symbol. The corresponding OFDM time-domain symbol, $\boldsymbol{x}$, of the optimized frequency-domain symbol, $\boldsymbol{c}$, can be obtained by inverse fast Fourier transform (IFFT) with $\ell$-times oversampling, i.e., $\boldsymbol{x}=\operatorname{IFFT}_{\ell}(\boldsymbol{c})=\boldsymbol{A c}$, where the matrix $A \in \mathbb{C}^{\ell N \times N}$ is the first $N$ columns of the corresponding IFFT matrix.

The OFDM subcarriers are usually divided into three disjoint sets: data subcarriers, free subcarriers, and pilot subcarriers with cardinalities $d, f$, and $p$, respectively, so that $d+f+p=N$, where $N$ is the total number of subcarriers. For simplicity, we do not consider pilot subcarriers in this paper, although the results can be easily generalized to systems with pilot subcarriers. Let $S \in \mathbb{R}^{N \times N}$ be a diagonal matrix with $S_{i i}=1$ when the $i$ th subcarrier is reserved for data transmission and $S_{i i}=0$ otherwise. We are now ready to define three parameters responsible for OFDM's reliable performance: PAPR, EVM, and FCPO.

The PAPR is defined as the ratio of the peak power of the signal to its average power. Mathematically, the PAPR of an OFDM symbol can be written as:

$$
\operatorname{PAPR}=\max _{i=1, \ldots, \ell N} \frac{\left|x_{i}\right|^{2}}{\frac{1}{\ell N}|| \boldsymbol{x}||^{2}}
$$

where $x_{i}$ denotes the $i$ th entry of the vector $\boldsymbol{x}$. An efficient way to reduce the PAPR is by distorting the OFDM constellation $[13,15]$. The level of distortion, measured by the EVM, should be kept at a minimum since a larger EVM value leads to a bit error rate (BER) performance degradation. A single OFDM symbol's EVM is mathematically defined as:

$$
\mathrm{EVM}=\frac{\sum_{i=i_{1}}^{i_{d}}\left|c_{i}-c_{0 i}\right|^{2}}{\sum_{i=i_{1}}^{i_{d}}\left|c_{0 i}\right|^{2}}=\frac{\left\|\boldsymbol{S}\left(\boldsymbol{c}-\boldsymbol{c}_{0}\right)\right\|^{2}}{\left\|\boldsymbol{S} \boldsymbol{c}_{0}\right\|^{2}},
$$

where the data subcarriers are indexed by $i_{1}, i_{2}, \ldots, i_{d}$. The PAPR can be further reduced by assigning a portion of energy to the free subcarriers $[13,15]$. The FCPO measures the value of free subcarriers' power and is defined as:

$$
\mathrm{FCPO}=\frac{\left\|\left(\boldsymbol{I}_{N}-S\right) \boldsymbol{c}\right\|^{2}}{\|\boldsymbol{S c}\|^{2}} .
$$

The FCPO should be kept small since it measures the fraction of power 'wasted' in the free subcarriers, which are not used to carry information. In this paper, we will focus on minimizing EVM for given PAPR and FCPO thresholds, i.e.,

$$
\underset{c \in \mathbb{C}^{N}}{\operatorname{minimize}} \mathrm{EVM}
$$

subject to

$$
\mathrm{PAPR} \leq \alpha,
$$

$$
\mathrm{FCPO} \leq \beta,
$$

where $\alpha$ and $\beta$ are PAPR and FCPO thresholds, respectively. It is straightforward to see that the optimization problem (4) to (6) is equivalent to (see also [16]):

$$
\underset{c \in \mathbb{C}^{N}}{\operatorname{minimize}}\left\|\boldsymbol{S}\left(\boldsymbol{c}-\boldsymbol{c}_{0}\right)\right\|^{2}
$$

subject to

$$
\begin{aligned}
& \boldsymbol{c}^{H}\left(\boldsymbol{M}_{i}-\boldsymbol{P}_{\alpha}\right) \boldsymbol{c} \leq 0, i=1, \ldots, \ell N, \\
& \boldsymbol{c}^{H}\left(\boldsymbol{I}_{N}-\boldsymbol{S}_{\beta}\right) \boldsymbol{c} \leq 0,
\end{aligned}
$$

where $\boldsymbol{M}_{i}=\ell N \boldsymbol{A}^{H} \boldsymbol{e}_{i} \boldsymbol{e}_{i}^{T} \boldsymbol{A}, \boldsymbol{P}_{\alpha}=\alpha \boldsymbol{A}^{H} \boldsymbol{A}, \boldsymbol{S}_{\beta}=(\beta+1) \boldsymbol{S}$ and $\boldsymbol{e}_{i}$ represents the $i$ th column of the identity matrix $\boldsymbol{I}_{\ell N}$. The EVM optimization framework (7) to (9) results in a nonconvex optimization problem since the matrices $\boldsymbol{I}_{N}-\boldsymbol{S}_{\beta}$ and $\boldsymbol{M}_{i}-\boldsymbol{P}_{\alpha}$, for $i=1, \ldots, \ell N$, are indefinite; in other words, all the constraints are nonconvex [17]. As most nonconvex problems, our problem is NP hard and, thus, difficult to solve [17].

\section{Optimization procedure}

In this section, we present an iterative method to generate optimized frequency-domain OFDM symbols. The method consists of solving a sequence of SOCP 
subproblems which approximate the original problem (7) to (9) locally. Let $\boldsymbol{c}_{(0)}$ be a randomly generated feasible starting point for the problem (7) to (9). A new feasible point $\boldsymbol{c}_{(1)}$ is obtained as the solution of the following program:

$$
\underset{c \in \mathbb{C}^{N}}{\operatorname{minimize}}\left\|\boldsymbol{S}\left(\boldsymbol{c}-\boldsymbol{c}_{0}\right)\right\|^{2}
$$

subject to

$$
\boldsymbol{c}^{H} \boldsymbol{M}_{i} \boldsymbol{c}-\boldsymbol{c}_{(0)}^{H} \boldsymbol{P}_{\alpha} \boldsymbol{c}_{(0)}-2 \Re\left(\boldsymbol{c}_{(0)}^{H} \boldsymbol{P}_{\alpha}\left(\boldsymbol{c}-\boldsymbol{c}_{(0)}\right)\right) \leq 0, \forall i
$$

$$
\boldsymbol{c}^{H} \boldsymbol{c}-\boldsymbol{c}_{(0)}^{H} \boldsymbol{S}_{\beta} \boldsymbol{c}_{(0)}-2 \Re\left(\boldsymbol{c}_{(0)}^{H} \boldsymbol{S}_{\beta}\left(\boldsymbol{c}-\boldsymbol{c}_{(0)}\right)\right) \leq 0,
$$

where $\Re\{z\}$ denotes the real part of the complex number $z$. The problem in (10) to (12) is obtained by linearizing the concave parts of the constraint functions in (8) to (9) around $\boldsymbol{c}_{(0)}$. (Note that this is actually the first-order Taylor expansion). This leads to a SOCP problem that can be readily solved by CVX [18]. The method continues with linearizing the original problem around $\boldsymbol{c}_{(1)}$ and repeating this procedure until convergence is reached. A justification/motivation of this reformulation lies in the fact that the best convex approximation of a concave function is an affine function.

This method generates a sequence of feasible points with nonincreasing objective values. To prove this claim, we proceed as follows. Note that (11) with $c=\boldsymbol{c}_{(1)}$ can be equivalently written as:

$$
\boldsymbol{c}_{(1)}^{H} \boldsymbol{M}_{i} \boldsymbol{c}_{(1)}+\boldsymbol{c}_{(0)}^{H} \boldsymbol{P}_{\alpha} \boldsymbol{c}_{(0)}-\boldsymbol{c}_{(0)}^{H} \boldsymbol{P}_{\alpha} \boldsymbol{c}_{(1)}-\boldsymbol{c}_{(1)}^{H} \boldsymbol{P}_{\alpha} \boldsymbol{c}_{(0)} \leq 0 .
$$

Now, note that

$$
\left(\boldsymbol{c}_{(1)}-\boldsymbol{c}_{(0)}\right)^{H} \boldsymbol{P}_{\alpha}\left(\boldsymbol{c}_{(1)}-\boldsymbol{c}_{(0)}\right) \geq 0,
$$

since $\boldsymbol{P}_{\alpha}$ is a positive semidefinite matrix. Thus, we can write

$$
\begin{aligned}
\left(\boldsymbol{c}_{(1)}-\right. & \left.\boldsymbol{c}_{(0)}\right)^{H} \boldsymbol{P}_{\alpha}\left(\boldsymbol{c}_{(1)}-\boldsymbol{c}_{(0)}\right) \geq \boldsymbol{c}_{(1)}^{H} \boldsymbol{M}_{i} \boldsymbol{c}_{(1)}+ \\
& +\boldsymbol{c}_{(0)}^{H} \boldsymbol{P}_{\alpha} \boldsymbol{c}_{(0)}-\boldsymbol{c}_{(0)}^{H} \boldsymbol{P}_{\alpha} \boldsymbol{c}_{(1)}-\boldsymbol{c}_{(1)}^{H} \boldsymbol{P}_{\alpha} \boldsymbol{c}_{(0)} .
\end{aligned}
$$

It is now straightforward to see that

$$
\boldsymbol{c}_{(1)}^{H}\left(\boldsymbol{M}_{i}-\boldsymbol{P}_{\alpha}\right) \boldsymbol{c}_{(1)} \leq 0,
$$

i.e., $\boldsymbol{c}_{(1)}$ satisfies the constraints in (8). It can be easily shown that $\boldsymbol{c}_{(1)}$ also satisfies the constraint in (9). To this aim, we combine the inequality

$$
\left(\boldsymbol{c}_{(1)}-\boldsymbol{c}_{(0)}\right)^{H} \boldsymbol{S}_{\beta}\left(\boldsymbol{c}_{(1)}-\boldsymbol{c}_{(0)}\right) \geq 0
$$

with (12) when $\boldsymbol{c}=\boldsymbol{c}_{(1)}$. Then, after some basic manipulations, we obtain $\boldsymbol{c}_{(1)}^{H}\left(\boldsymbol{I}_{N}-\boldsymbol{S}_{\beta}\right) \boldsymbol{c}_{(1)} \leq 0$, i.e., $\boldsymbol{c}_{(1)}$ satisfies the constraint in (9). Thus, $\boldsymbol{c}_{(1)}$ is feasible for the original problem (7) to (9).

Next, remark that $\boldsymbol{c}_{(0)}$ is feasible for (10) to (12) (when $c=c_{(0)}$, the constraints (8) and (9) become identical to (11) and (12), respectively). This implies that the feasible set of (10) to (12) contains $\boldsymbol{c}_{(0)}$. Thus, $\left\|\boldsymbol{S}\left(\boldsymbol{c}_{(1)}-\boldsymbol{c}_{0}\right)\right\|^{2} \leq\left\|\boldsymbol{S}\left(\boldsymbol{c}_{(0)}-\boldsymbol{c}_{0}\right)\right\|^{2}$, i.e., the objective value of the new feasible point $\boldsymbol{c}_{(1)}$ cannot be higher than the objective value of the starting point $\boldsymbol{c}_{(0)}$. The algorithm stops when $\left\|\boldsymbol{S}\left(\boldsymbol{c}_{(k)}-\boldsymbol{c}_{0}\right)\right\|-\left\|\boldsymbol{S}\left(\boldsymbol{c}_{(k+1)}-\boldsymbol{c}_{0}\right)\right\|<10^{-4}$ for some $k$. We refer to the proposed method as 'NEW SOCP' from hereafter.

Naturally, the algorithm requires a strict feasible solution of (7) to (9) as the feasible starting point. The problem of finding a feasible solution is not a trivial task and, unfortunately, no solid theory is available. However, the following heuristic has shown to be efficient in addressing this problem. The feasible starting point $\boldsymbol{c}_{(0)}$ is obtained as a solution of the convex feasibility problem:

$$
\underset{c \in \mathbb{C}^{N}}{\text { find }} \boldsymbol{c}
$$

subject to

$$
\begin{aligned}
& \boldsymbol{c}^{H} \boldsymbol{M}_{i} \boldsymbol{c}-f_{1}-2 \Re\left(\boldsymbol{c}_{(\mathrm{rand})}^{H} \boldsymbol{P}_{\alpha}\left(\boldsymbol{c}-\boldsymbol{c}_{(\mathrm{rand})}\right)\right) \leq 0, \forall i \\
& \boldsymbol{c}^{H} \boldsymbol{c}-f_{2}-2 \Re\left(\boldsymbol{c}_{(\mathrm{rand})}^{H} \boldsymbol{S}_{\beta}\left(\boldsymbol{c}-\boldsymbol{c}_{(\mathrm{rand})}\right)\right) \leq 0,
\end{aligned}
$$

where $f_{1}=\boldsymbol{c}_{\text {(rand) }}^{H} \boldsymbol{P}_{\alpha} \boldsymbol{c}_{\text {(rand) }}, f_{2}=\boldsymbol{c}_{\text {(rand) }}^{H} \boldsymbol{S}_{\beta} \boldsymbol{c}_{(\text {rand })}$ and $\boldsymbol{c}_{(\text {rand })}$ is randomly generated; the real and imaginary parts of the $i$ th entry of the vector $\boldsymbol{c}_{(\text {rand) }}$ are randomly generated in the intervals $\left[\Re\left\{c_{0 i}\right\}-1, \Re\left\{c_{0 i}\right\}+1\right]$ and $\left[\Im\left\{c_{0 i}\right\}-1, \Im\left\{c_{0 i}\right\}+1\right]$, respectively, where $c_{0 i}$ is the $i$ th entry of the vector $c_{0}$ and $\Im\{a\}$ denotes the imaginary part of the complex number $a$.

Problem (13) to (15) is obtained from (10) to (12) with $\boldsymbol{c}_{0}=\boldsymbol{c}_{\text {(rand) }}$ and can be solved using CVX. Problem (13) to (15) may be infeasible; however, when it is feasible, this heuristic will provide a feasible solution to problem (7) to (9) which is used as the starting point of the algorithm ${ }^{\mathrm{a}}$.

It is important to note that the existing convex methods $[15,16]$ may fail to deliver feasible solution to the EVM minimization problem (4) to (6); please see ([15], Sec. III-C), and ([16], Sec. IV). This is due to the fact that in $[15,16]$, the feasible (nonconvex) set of the original (nonconvex) problem (4) to (6) lies within the feasible (convex) set of the relaxed convex problem. Note that in our work, in sharp contrast to $[15,16]$, the feasible set of the relaxed convex problem is a convex subset of the original (nonconvex) feasible set. Hence, the feasibility of the new solution is guaranteed. 
Note: We can introduce an optional constraint that will keep the EVM below some preset threshold. This corresponds to a more challenging and realistic scenario where PAPR, FCPO, and EVM are simultaneously constrained. In that case, the EVM optimization can be formulated as

$$
\underset{p \in \mathbb{R}, c \in \mathbb{C}^{N}}{\operatorname{minimize}} p
$$

subject to

$$
\mathrm{EVM} \leq p \mathrm{EVM}_{\max }
$$

$$
\text { (5), (6), } p \leq 1
$$

where $\mathrm{EVM}_{\max }$ is the maximum allowed EVM. Note that the optimization problem (4) to (6) is different from the one in (16) to (18), since the search space for $c$ in the former is larger than that in the latter.

We remark that the constraint (17) is convex and, consequently, the EVM problem (16) to (18) can also be addressed by the proposed method ${ }^{\mathrm{b}}$. The simulation results in Section 4 will assess the effectiveness of the new method.

\section{Performance results}

Extensive simulations were performed to compare the performance of the proposed algorithm with existing algorithms. Unless stated otherwise, the number of subcarriers is $N=64$, the number of data subcarriers is $d=$ 52 and the number of free subcarriers is $f=12$. The data subcarriers were generated from 16-quadrature amplitude modulation (QAM), and 64-QAM constellations and the oversampling factor was assumed to be $\ell=4$. The CVX package [18] for specifying and solving convex programs was used to solve (10) to (12).

To evaluate the performance of the proposed technique and compare it with existing ones, we obtained the complementary cumulative density function (CCDF) of the PAPR, which corresponds to the probability that the PAPR of an arbitrary OFDM symbol exceeds a given threshold.

The performance of the new algorithm, denoted here by 'NEW SOCP', will be compared with the performance of the SDP-based approach proposed in [16], denoted here by 'SDP WANG', iterative SOCP-based approach presented in [15], denoted here by 'SOCP WANG', and RCF (four iterations) [7], denoted here by 'RCF'.

For the sake of presentation simplicity, we divide the comparison in two cases: without EVM constraint and with EVM constraint.

\subsection{Without EVM constraint}

Figures 1 and 2 show the CCDFs of the PAPR of modified symbols using the new and existing algorithms for 16-QAM OFDM and 64-QAM OFDM, respectively. The PAPR is set to 4 and $4.5 \mathrm{~dB}$ for 16-QAM OFDM and 64-QAM OFDM, respectively, and the FCPO is set to 0.15 .

From Figures 1 and 2, we can observe that all of the methods can reduce the PAPR. We see that the new approach and the existing convex method, SDP WANG,

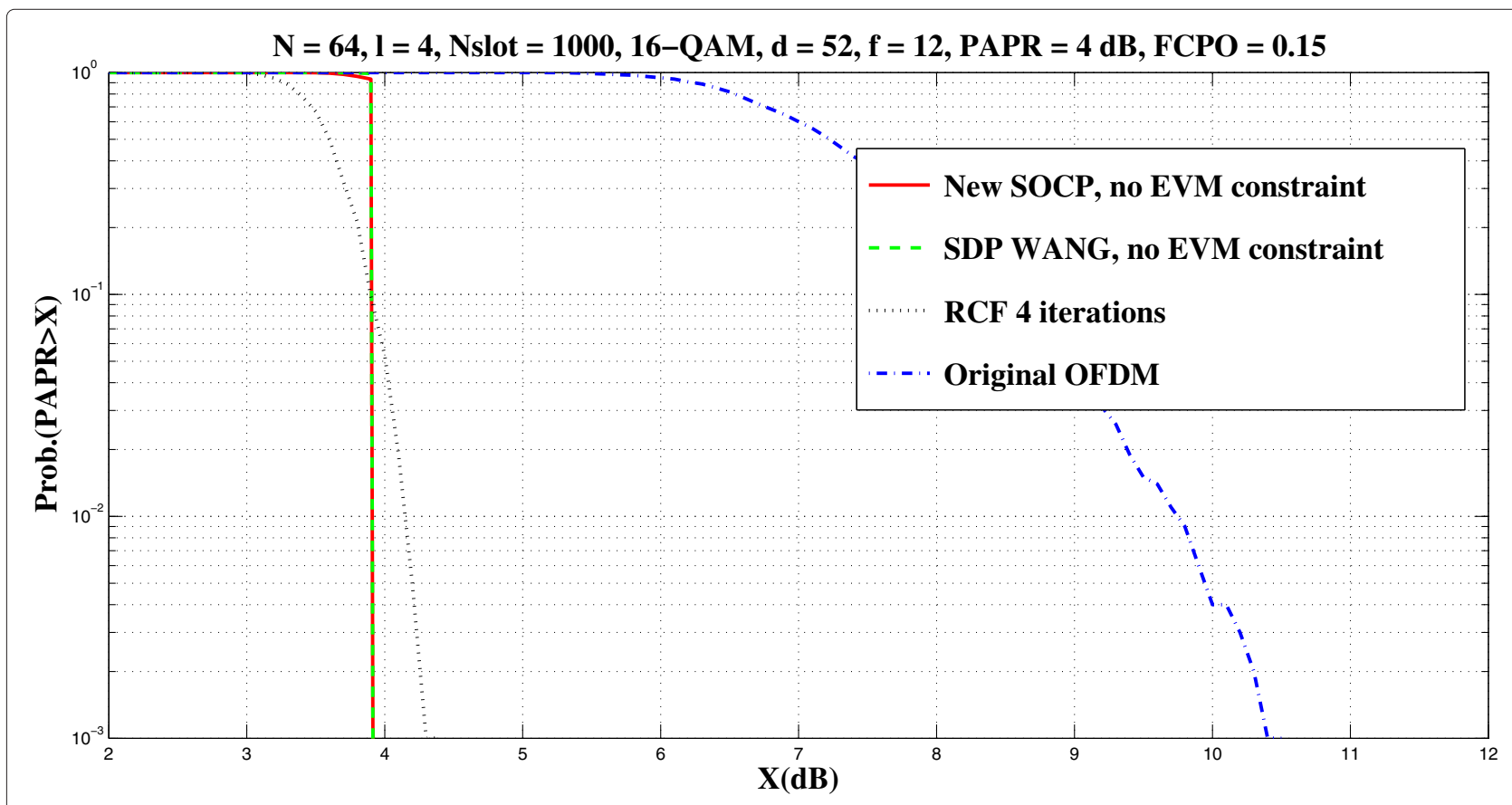

Figure 1 Comparisons of PAPR CCDF curves: 16-QAM, without EVM constraint. 


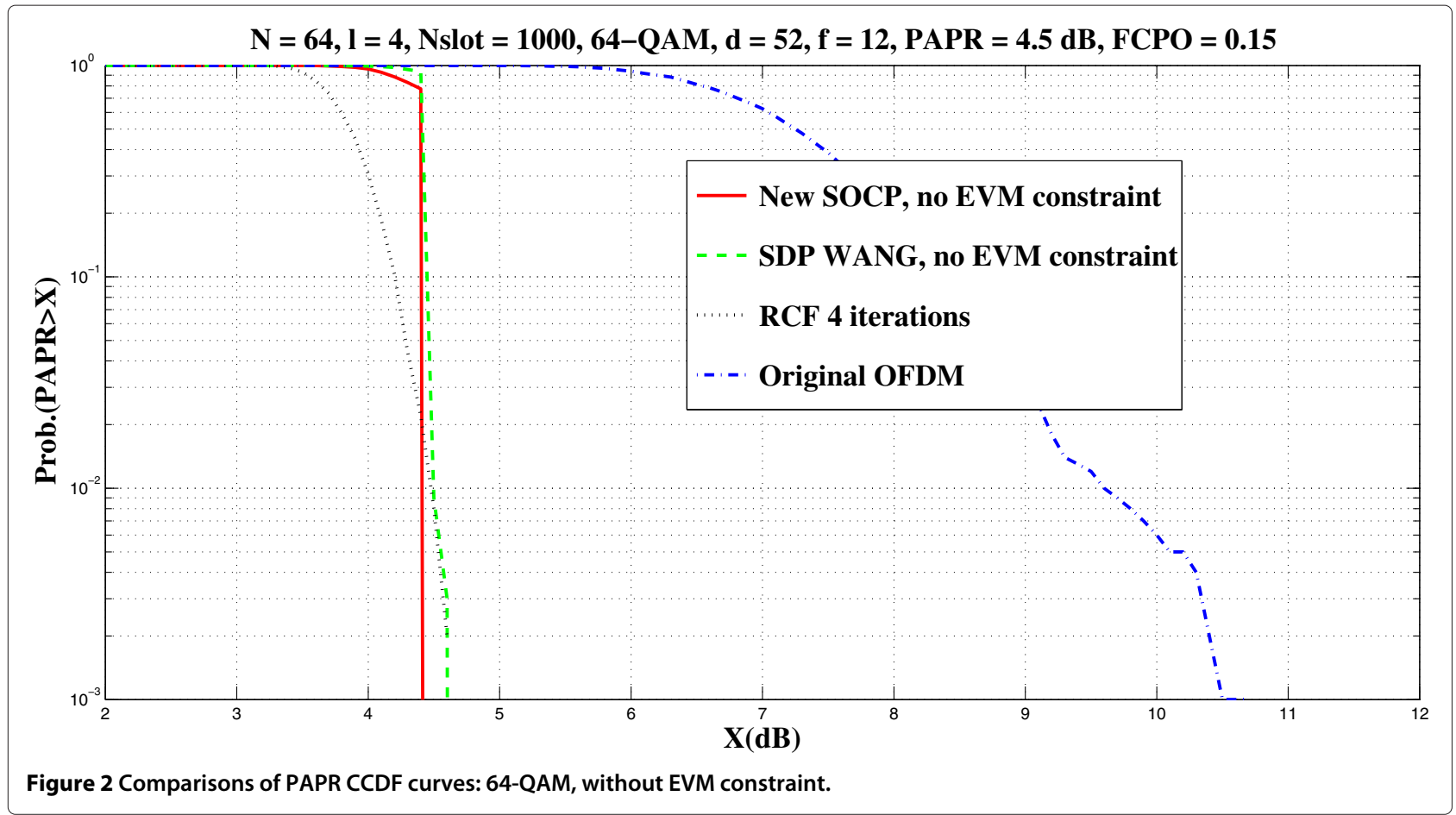

show almost identical performance, with their CCDF curves having sharp cutoff at $4 \mathrm{~dB}$ for 16-QAM and $4.5 \mathrm{~dB}$ for 64-QAM. When compared to 'RCF', the new approach has somewhat sharper cutoff, indicating reduced variation in the PAPR of our optimized symbols. It is important to point out that the new approach was successful in finding a feasible solution at each simulation run. This was however not the case with SDP WANG since it sometimes breaks the PAPR constraint for 64-QAM.

Figures 3 and 4 plot the BER versus $E_{b} / N_{0}$ curves for, respectively, 16-QAM OFDM and 64-QAM OFDM in AWGN channels, where $E_{b}$ denotes the average bit energy

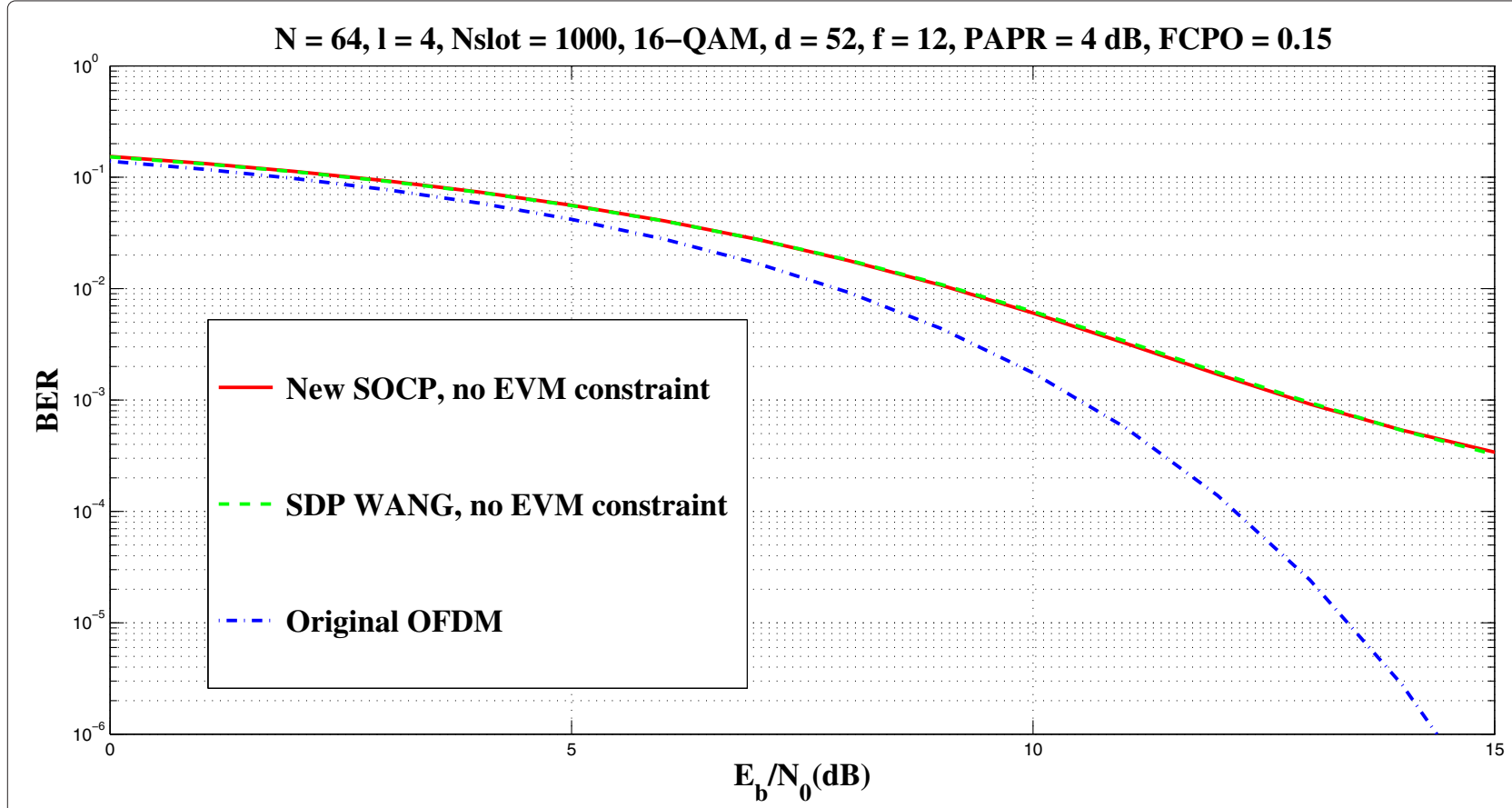

Figure 3 BER comparisons: 16-QAM, without EVM constraint. 


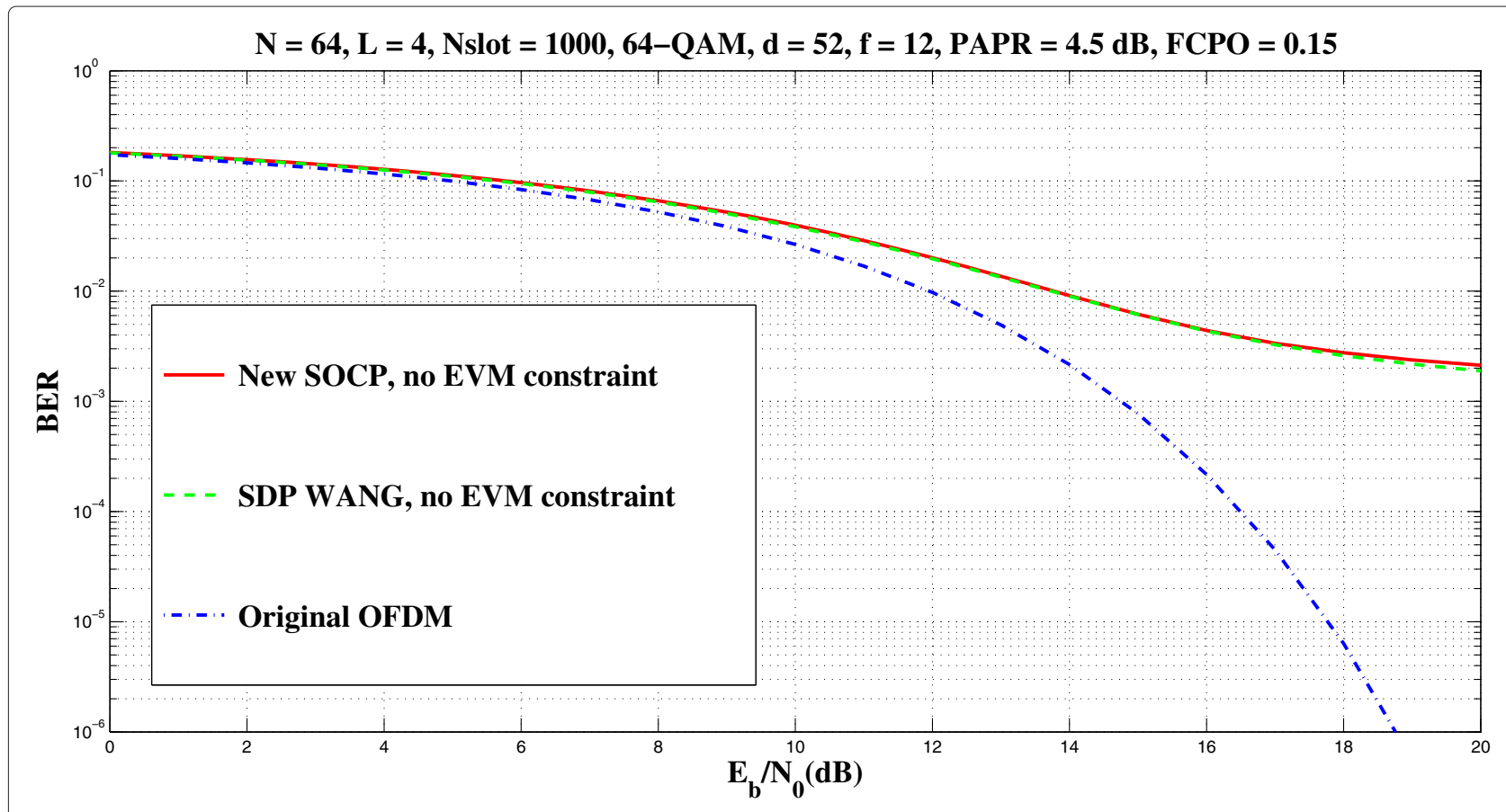

Figure 4 BER comparisons: 64-QAM, without EVM constraint.

and $N_{0}$ is the one-sided power spectral density of the noise component. We see that NEW SOCP and SDP WANG have similar BER performance.

\subsection{With EVM constraint}

Figures 5 and 6 plot the CCDFs of the PAPR of modified symbols using the new and existing algorithms for
16-QAM OFDM and 64-QAM OFDM, respectively. The PAPR is set to 4 and $4.5 \mathrm{~dB}$ for 16-QAM OFDM and 64QAM OFDM, respectively, the EVM constraint is set to 0.09 and 0.04 for 16-QAM OFDM and 64-QAM OFDM, respectively, and the FCPO is set to 0.15 .

From Figures 5 and 6, we can observe that all of the methods can reduce the PAPR. We also see that the

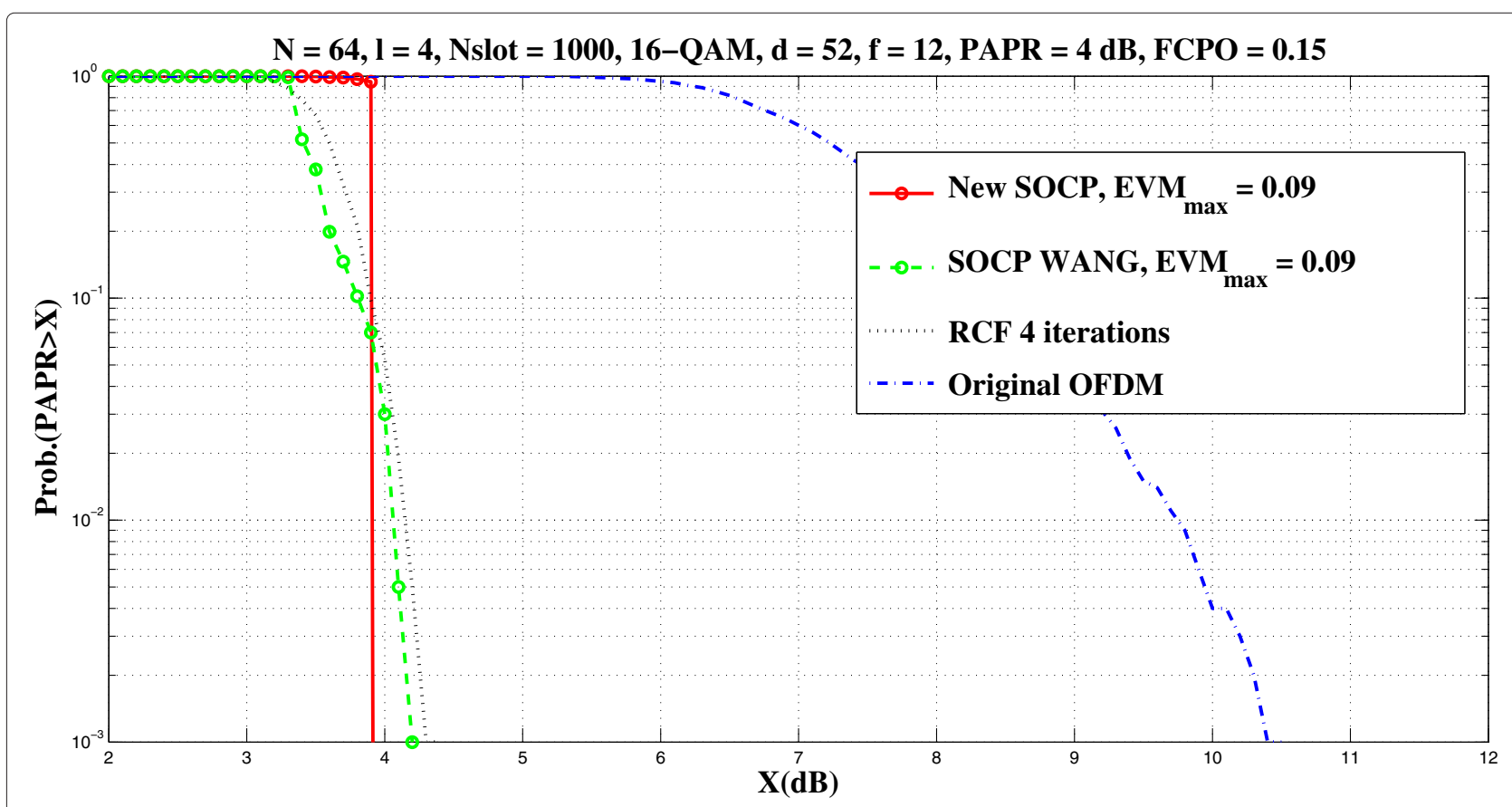

Figure 5 Comparisons of PAPR CCDF curves: 16-QAM, with EVM constraint. 


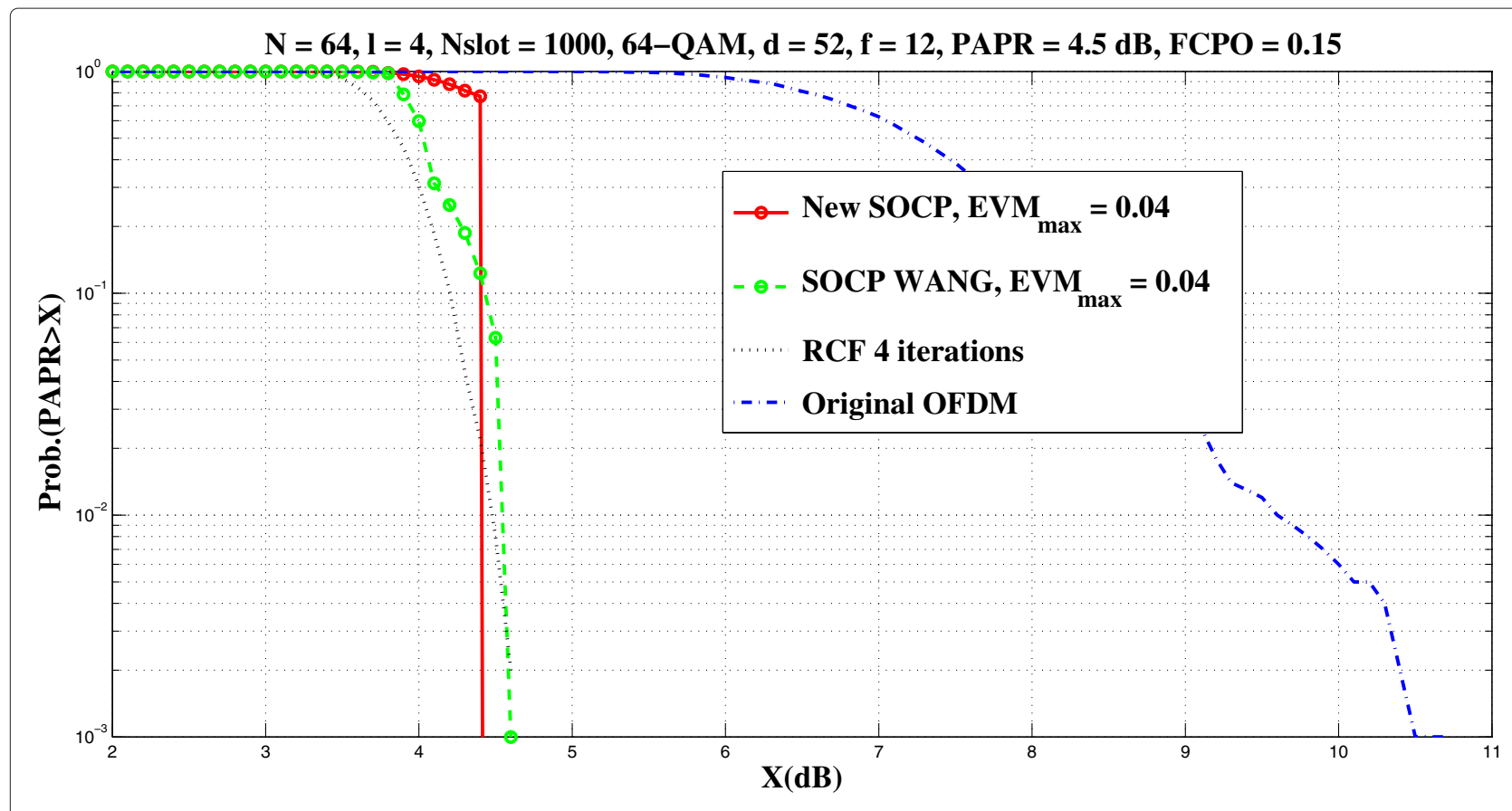

Figure 6 Comparisons of PAPR CCDF curves: 64-QAM, with EVM constraint.

new approach maintains sharp cutoff, whereas the CCDF curve of the state-of-the-art convex approach, SOCP WANG, has a more gradual cutoff.

Figures 7 and 8 show the BER versus $E_{b} / N_{0}$ curves for, respectively, 16-QAM OFDM and 64-QAM OFDM in
AWGN channels. We see that NEW SOCP outperforms significantly SOCP WANG when $\mathrm{EVM}_{\max }=0.04$ and $E_{\text {VMax }}=0.09$. We also observe that NEW SOCP for $\mathrm{EVM}_{\max }=0.04$ has the best BER performance ${ }^{\mathrm{c}}$. This demonstrates that our optimized symbols for $\mathrm{EVM}_{\max }=$

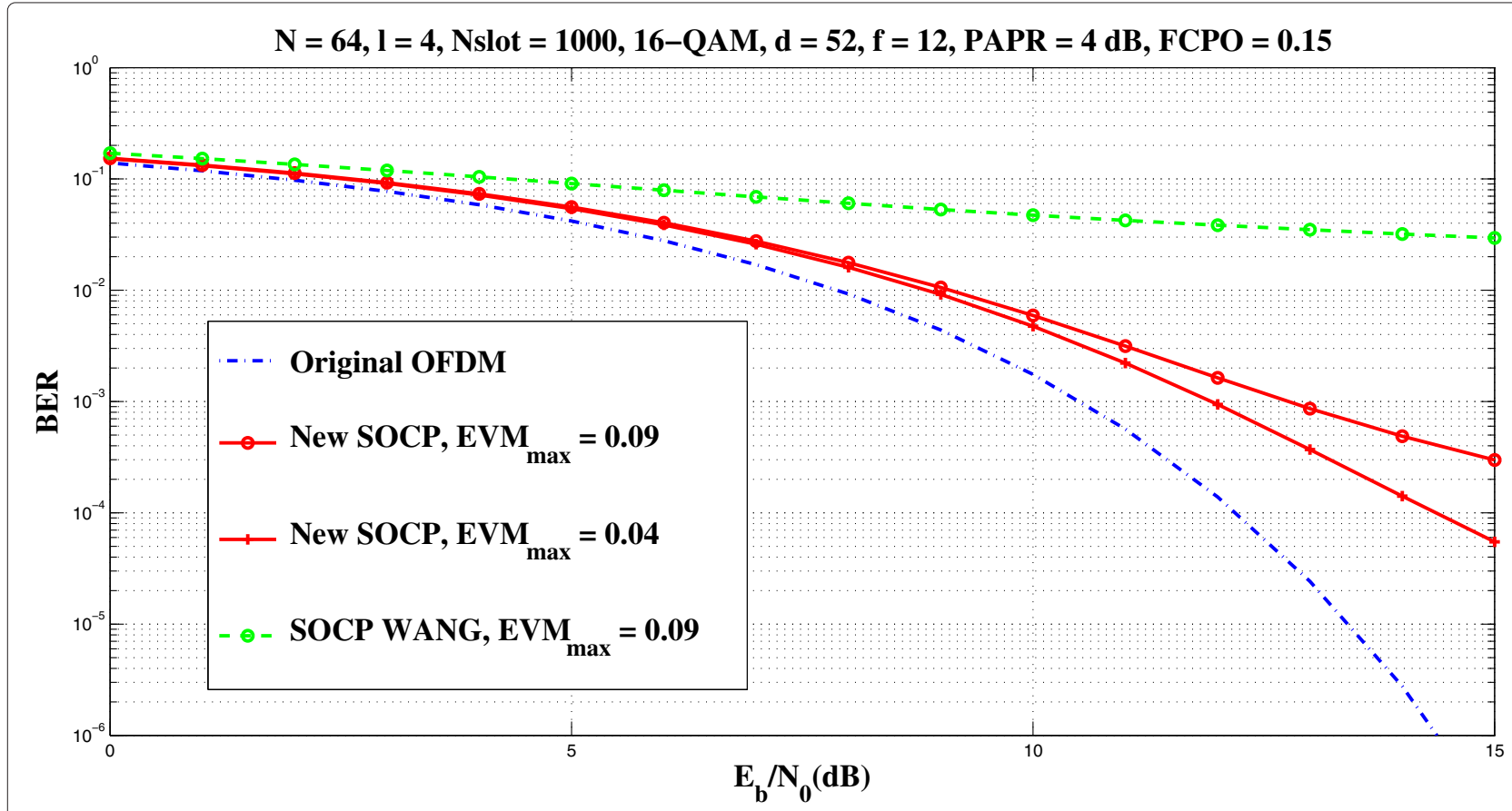

Figure 7 BER comparisons: 16-QAM, with EVM constraint. 


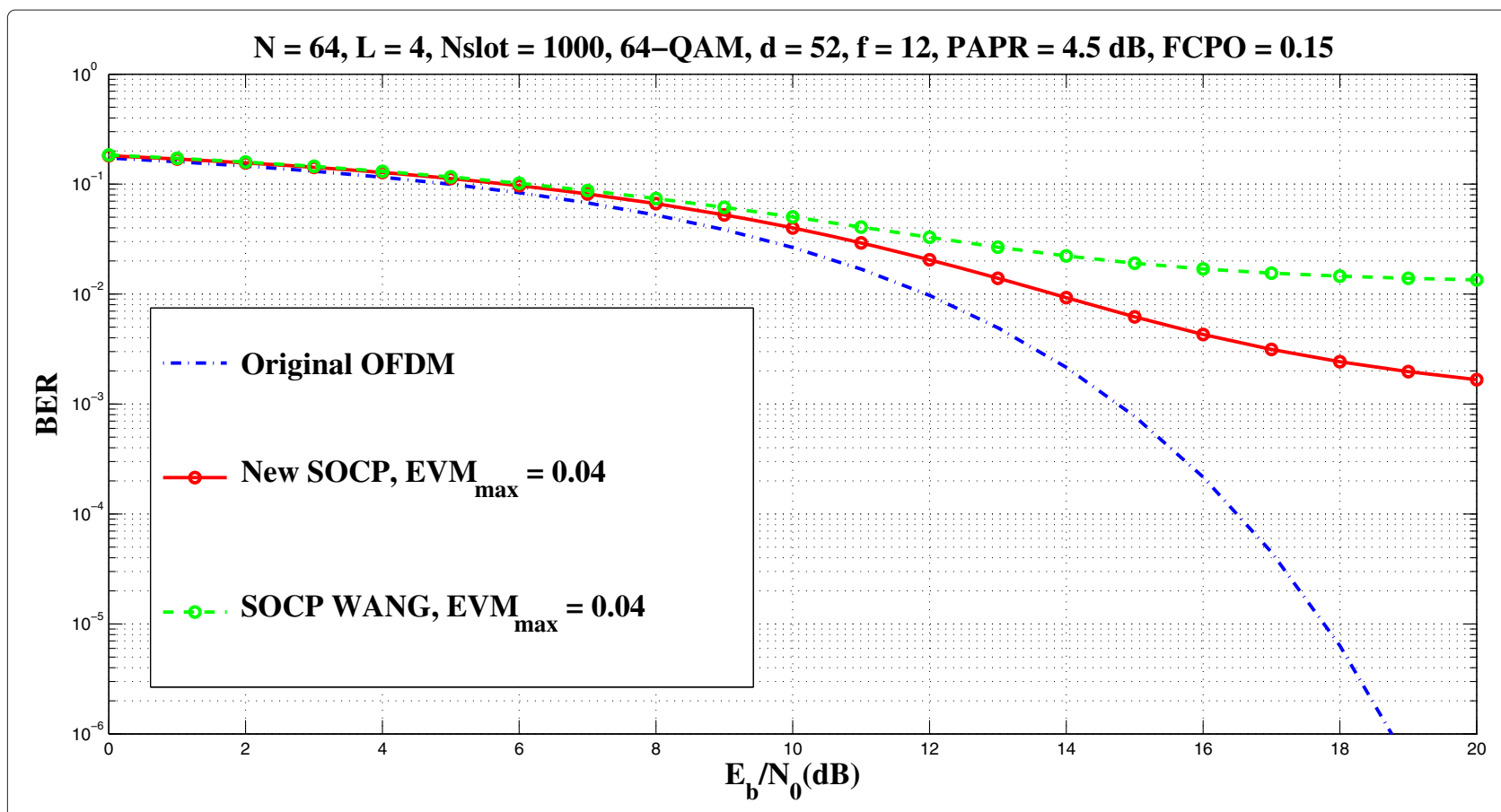

Figure 8 BER comparisons: 64-QAM, with EVM constraint.

0.04 have less distortion than those of symbols obtained by SOCP WANG and NEW SOCP, the latter two with $\mathrm{EVM}_{\text {max }}=0.09$.

Tables 1 and 2 display the percentages of infeasible solutions provided by the new approach and SOCP WANG for the 16-QAM and 64-QAM modulation formats, respectively. The feasibility issue is important since we have found that both of the two approaches may fail to produce feasible solution to the EVM problem (16) to (18). Note that only successful trials were presented in Figures 5,6,7 and 8.

From the comparisons presented in Tables 1 and 2, it can be seen that the probability of generating infeasible solutions by the new approach is reduced when compared to SOCP WANG. Furthermore, as expected, for a given modulation scheme, this probability decreases as the preset threshold $E M_{\text {max }}$ increases since the search space defined by the constraints (17) to (18) becomes larger. As a final note, remark that it is an open question whether for a given set of system parameters $\left(\mathrm{EVM}_{\max }, \alpha, \beta, \boldsymbol{c}_{0}, N, d\right.$, $f$ ) a feasible solution to the EVM problem actually exists.

Table 1 Infeasibility analysis for 16-QAM

\begin{tabular}{lcc}
\hline 16-QAM & EVM $_{\text {max }}=\mathbf{0 . 0 4}$ & EVM $_{\text {max }}=\mathbf{0 . 0 9}$ \\
\hline NEW SOCP & 19.29 & 2.44 \\
SOCP WANG & 40.41 & 38.40 \\
\hline
\end{tabular}

Note: The percentage of infeasible solutions obtained using the new approach and the Wang iterative SOCP approach [15]. PAPR $=4 \mathrm{~dB}$ and FCPO $=0.15$.
Figures 9 and 10 display the optimized frequencydomain OFDM symbols obtained using NEW SOCP, with the right subplots showing only the data subcarriers, for the cases when the EVM constraint is not active and when the EVM constraint is active with $\mathrm{EVM}_{\max }=0.04$, respectively. We observe that, as expected, the clusters around the constellation points are more compact when the EVM constraint is active. We also see that the new approach assigns power to the free subcarriers.

\subsection{Complexity analysis}

The comparison of computational complexity is also of interest. Table 3 shows the worst-case complexities of the new and existing approaches. The new approach is iterative, and its complexity increases linearly with the number of iterations $L$. During the simulations, we observed that a small number of iterations (e.g., $L=10$ ) is sufficient to ensure the convergence of the algorithm. From Table 3, we see that when the EVM constraint is not active, the complexity of the new approach is lower than that of SDP WANG and higher than that of RCF. In the case when the

Table 2 Infeasibility analysis for 64-QAM

\begin{tabular}{lcc}
\hline 64-QAM & EVM $_{\text {max }}=\mathbf{0 . 0 4}$ & EVM $_{\text {max }}=\mathbf{0 . 0 9}$ \\
\hline NEW SOCP & 1.67 & 0.20 \\
SOCP WANG & 42.57 & 32.84 \\
\hline
\end{tabular}

Note: The percentage of infeasible solutions obtained using the new approach and the Wang iterative SOCP approach [15]. PAPR $=4.5 \mathrm{~dB}$ and FCPO $=0.15$. 

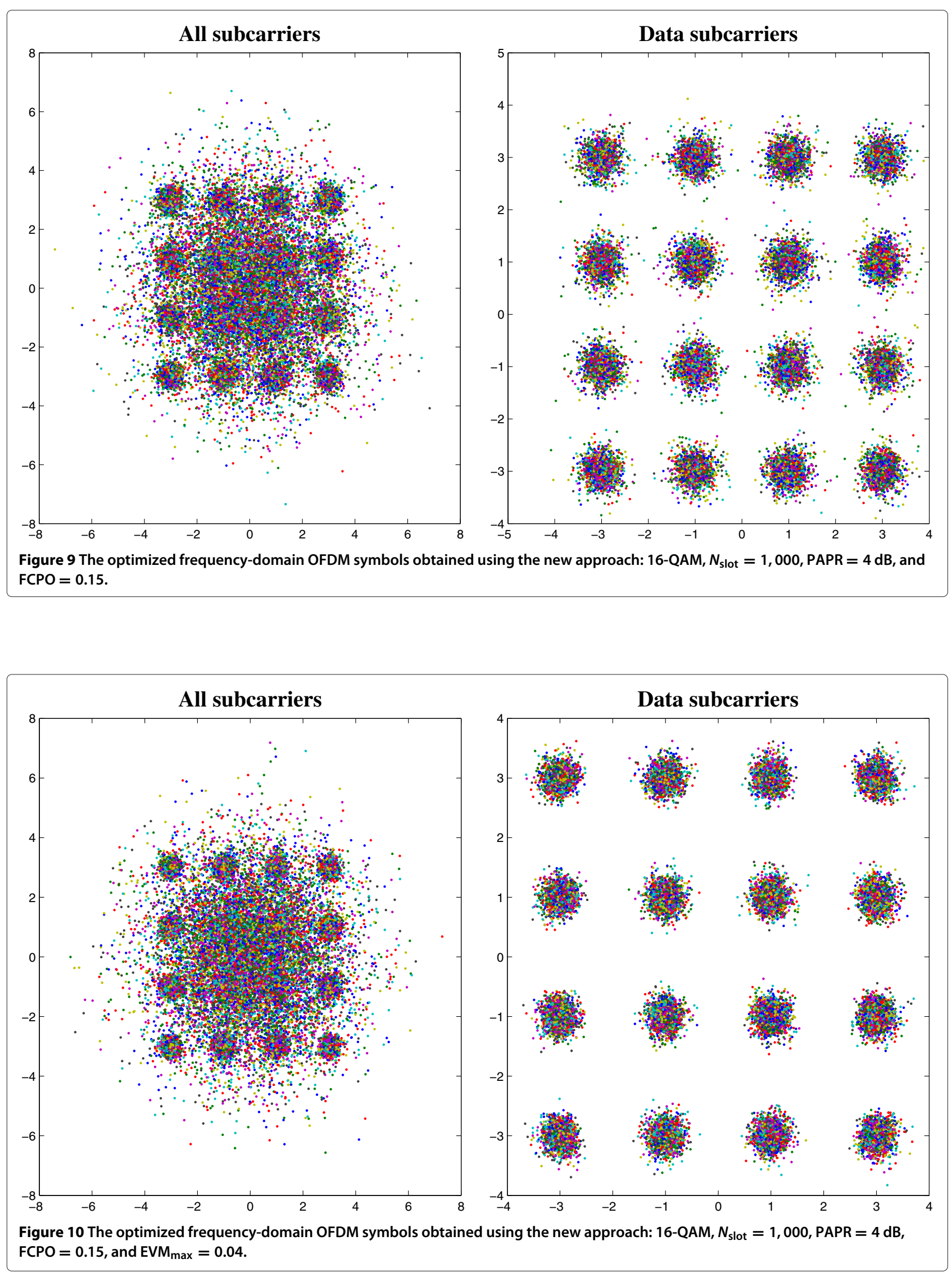
Table 3 Computational complexity analysis

\begin{tabular}{lccc}
\hline NEW SOCP & SDP WANG & SOCP WANG & RCF \\
\hline$O\left(L N^{3.5} \ell^{1.5}\right)$ & $O\left(N^{4.5} \ell^{2}\right)$ & $O\left(L N^{3.5} \ell^{1.5}\right)$ & $O(2 L \ell N \log (\ell N))$ \\
\hline
\end{tabular}

Note: $L$ is the maximum iteration number.

EVM constraint is active, the worst-case complexity of the new approach is the same as that of SOCP WANG. See [19] for more details about complexity issues. We see that the complexity of a convex-based approach is higher than that of RCF. However, it is important to know what are upper abounds on the achievable performance, e.g., in the case when all the three system parameters are constrained simultaneously. And this is exactly what the new method provides.

\section{Conclusions}

We have revisited the PAPR problem in OFDM systems. We formulated the problem as a nonconvex optimization problem which is solved approximately by an efficient iterative method. The simulation results show that in the case when the EVM constraint is not active, the new method outperforms the one in [16] in terms of complexity and feasibility, whereas, in the case when a constraint on the EVM is imposed, the new method outperforms the method in [15] in terms of PAPR performance, BER performance, and feasibility. This confirms the relevance of the approach proposed herein.

\section{Endnotes}

${ }^{a}$ It is not difficult to see that the solution of (13) to (15), $\boldsymbol{c}_{(0)}$, is feasible for the original problem (7) to (9).

${ }^{\mathrm{b}}$ In [13], Aggarwal and Meng proposed to minimize the PAPR subject to constraints on the EVM and FCPO. Note that the proposed approach can be readily applied to this framework as well.

'It was shown in [16] that 'RCF' exhibits a bad BER performance. Consequently, the BER performance of 'RCF' was not presented in Figures 3, 4, 7 and 8.

\section{Competing interests}

The authors declare that they have no competing interests.

\section{Acknowledgements}

This work was partially supported by Fundação para a Ciência e a Tecnologia under Projects PTDC/EEI-TEL/2990/2012-ADIN,

EXPL/EEI-TEL/1582/2013-GLANC, PEst-OE/EEI/UI0066/2014 and

EXPL/EEI-TEL/0969/2013-MANY2COMWIN, and Ciência 2008 Post-Doctoral Research grant. M. Beko is a collaborative member of INESC-INOV, Instituto Superior Técnico, University of Lisbon, Lisbon, Portugal.

\footnotetext{
Author details

${ }^{1}$ Universidade Lusófona de Tecnologias e Humanidades, Campo Grande, Lisbon 1749-024, Portugal. ${ }^{2}$ UNINOVA, Campus FCT/UNL, Monte de Caparica 2825-114, Portugal. ${ }^{3}$ Faculdade de Ciências e Tecnologia, Universidade Nova de Lisboa, Campus FCT/UNL, Monte de Caparica 2829-516, Portugal. ${ }^{4}$ Instituto de Telecomunicações, Torre Norte, Av. Rovisco Pais, Lisbon 1049-001, Portugal. ${ }^{5}$ University of Donja Gorica, Donja Gorica bb, Podgorica 81000, Montenegro.
}

Received: 14 May 2014 Accepted: 8 August 2014

Published: 21 August 2014

\section{References}

1. R Nee, R Prasad, OFDM for Wireless Multimedia Communications. (Artech House, Norwood, 2000)

2. SH Han, JH Lee, An overview of peak-to-average power ratio reduction techniques for multicarrier transmission. IEEE Wireless Commun. 12(2), 56-65 (2005)

3. A Jones, T Wilkinson, Combined coding for error control and increased robustness to system nonlinearities in OFDM, in IEEE VTC'96 (IEEE, Atlanta 1996)

4. S Müller, R Bräuml, R Fisscher, J Huber, OFDM with reduced peak-to-average power ratio by multiple signal representation. Annales Telecommun. 52, 58-67 (1997)

5. L Cimini Jr, N Sollenberger, Peak-to-average power reduction of an OFDM signal using partial transmit sequences. IEEE Comm. Lett. 4(3), 86-88 (1999)

6. R Dinis, A Gusmão, On the performance evaluation of OFDM transmission using clipping techniques, in IEEE VTC'99 (Fall) (IEEE, Amsterdam, 1999)

7. J Armstrong, Peak-to-average power reduction for OFDM by repeated clipping and frequency domain filtering. Electron. Lett. 38(5), 246-247 (2002)

8. R Dinis, A Gusmão, A class of nonlinear signal processing schemes for bandwidth-efficient OFDM transmission with low envelope fluctuation. IEEE Trans. Comm. 52(11), 2009-2018 (2004)

9. L-Q Wang, C Tellambura, Analysis of clipping noise and tone reservation algorithms for peak reduction in OFDM systems. IEEE Trans. Veh. Technol. 57(3), 1675-1694 (2008)

10. Y-C Wang, Z-Q Luo, Optimized iterative clipping and filtering for PAPR reduction of OFDM signals. IEEE Trans. Commun. 59(1), 33-37 (2011)

11. J Tellado, Peak to average power reduction for multicarrier modulation. Ph.D. dissertation, Stanford Univ., Stanford, CA, (2000)

12. B Krongold, $L$ Jones, An active-set approach for OFDM PAR reduction via tone reservation. IEEE Trans. Signal Process. 52(2), 495-509 (2004)

13. A Aggarwal, T Meng, Minimizing the peak-to-average power ratio of OFDM signals using convex optimization. IEEE Trans. Signal Process. 54(8), 3099-3110 (2006)

14. Q-J Liu, R Baxley, X Ma, G-T Zhou, Error vector magnitude optimization for OFDM systems with a deterministic peak-to-average power ratio constraint. IEEE J. Sel. Topics Signal Process. 3(3), 418-429 (2009)

15. Y-C Wang, $\mathrm{K}-\mathrm{C}$ Yi, Convex optimization method for quasi-constant peak-to-average power ratio of OFDM signals. IEEE Signal Process. Lett. 16(6), 509-512 (2009)

16. Y-C Wang, J-L Wang, K-C Yi, B Tian, PAPR reduction of OFDM signals with minimized EVM via semidefinite relaxation. IEEE Trans. Veh. Technol. 60(9), 4662-4667 (2011)

17. S Boyd, L Vandenberghe, Convex Optimization. (Cambridge University Press, New York, 2004)

18. M Grant, S Boyd. 'CVX: Matlab software for disciplined convex programming', version 1.21 (2010). http://cvxr.com/cvx

19. I Pólik, T Terlaky, Interior point methods for nonlinear optimization, in Nonlinear Optimization. 1st Edition, ed. by G Di Pillo, F Schoen (Springer, Berlin, 2010)

doi:10.1186/1687-6180-2014-130

Cite this article as: Beko et al: Peak reduction in OFDM using second-order cone programming relaxation. EURASIP Journal on Advances in Signal Processing 2014 2014:130 